\title{
H-388 0, HÍBRIDO DE MAÍZ TRILINEAL DE GRANO BLANCO OLEOSO PARA EL SUBTRÓPICO DE MÉXICO
}

\section{H-388 0, A THREE-WAY OILY WHITE-KERNEL MAIZE HYBRID FOR SUBTROPICS OF MEXICO}

\section{Ricardo E. Preciado-Ortiz *, Arturo D. Terrón Ibarra', Ma. Gricelda Vázquez-Carrillo², Noel O. Gómez-Montiel ${ }^{3}$, Dolores Briones-Reyes ${ }^{4}$ y Alfonso Peña-Ramos ${ }^{4}$}

\begin{abstract}
'Instituto Nacional de Investigaciones Forestales, Agrícolas y Pecuarias (INIFAP), Campo Experimental Bajío, Celaya, Guanajuato, México. ${ }^{2}$ INIFAP, Campo Experimental Valle de México, Texcoco, Estado de México, México. ${ }^{3}$ NIFAP, Campo Experimental Iguala, Iguala, Guerrero, México. ${ }^{4} I N I F A P$, Campo Experimental Pabellón, Pabellón de Arteaga, Aguascalientes, México.
\end{abstract}

*Autor de correspondencia (preciado.ernesto@inifap.gob.mx, repreciado@yahoo.com)

El maíz (Zea mays L.) en México es un insumo de platillos tradicionales, alimentos balanceados y productos industriales. Su diversidad genética permite, mediante diversos métodos de mejoramiento, obtener variedades con mayor valor agregado, tal es el caso de las variedades oleosas que producen granos con alto contenido de aceite (ACA), lo cual incrementa el valor de la cadena productiva de esta especie, en beneficio de los productores, industriales y consumidores. Un maíz se considera oleoso cuando su contenido de aceite es superior al 6 \% (Lambert, 2000). El H-388 O, híbrido de maíz oleoso de grano blanco, fue generado por el Programa de Maíz del INIFAP en el Campo Experimental Bajío (CEBAJ), como alternativa para la industria de la masa y la tortilla, ya que además de producir buen rendimiento de tortilla (1.5 $\mathrm{kg} \mathrm{kg}^{-1}$ de maíz), su mayor cantidad de ácidos grasos insaturados (oleico y linoleico) imparten mayor suavidad a las mismas; además, el mayor contenido calórico del grano puede mejorar la conversión alimenticia en la producción de huevo, carne y leche. También puede ser una excelente fuente de materia prima para el desarrollo de nuevos productos en la industria de la transformación (Alexander, 1988; Lambert, 2000).

El H-388 $\mathrm{O}$ es un híbrido trilineal, en el que interviene una cruza simple (LBDHBO $1 \times$ LBDHBO 2) como progenitor femenino y la línea LBDHBO 3 como progenitor masculino (Cuadro 1). En la cruza simple participan dos líneas doble haploides $(\mathrm{LDH})$ derivadas de la Población Blanca Bajío
(PBB); el progenitor masculino es una LDH derivada de la Población Blanca del Noroeste (PBN).

El germoplasma involucrado en la PBB proviene de la variedad $\vee 385$, que se obtuvo a partir del octavo ciclo de recombinación y selección para porte bajo de la Población de Amplia Base Genética Tardía (PABG T) del Bajío, la cual se integró con material básico y experimental, colectas y fuentes introducidas de origen subtropical. Por su parte, la PBN involucra germoplasma proveniente de híbridos y líneas con excelente adaptación al Noroeste, con resistencia a la roya de la hoja (Puccinia polysora) y con excelente calidad de tallo (Ortega-Corona et al., 2015). La razón de seleccionar de manera simultánea en la PBB y en la PBN fue para aprovechar la respuesta heterótica que se obtiene al cruzar ambas poblaciones y así generar híbridos ACA de alto potencial de rendimiento.

El incremento del contenido de aceite en las poblaciones PBB y PBN se inició en el año 2004, por medio de un esquema de selección recurrente de familias de medios hermanos, en lotes aislados, en los que se sembraron las 50 familias con mayor contenido de aceite del ciclo anterior. En cada ciclo se seleccionaron las cuatro mejores mazorcas dentro de cada familia, provenientes de plantas sanas, con competencia completa, sin acame y con altura de planta y mazorca que no excediera la media de la población. En laboratorio, el contenido de aceite se midió

Cuadro 1. Denominación, genealogía y obtentor de los progenitores que integran H-388 0 de grano blanco oleoso.

\begin{tabular}{llll}
\hline Parental & Denominación & Genealogía & Obtentor \\
\hline A & LBDHBO 1 & PBB DH 178 & INIFAP \\
B & LBDHBO 2 & PBB DH 183 & INIFAP \\
C & LBDHBO 3 & PBB DH 85 & INIFAP \\
\hline
\end{tabular}


inicialmente en un espectrofotómetro de rayos infrarrojos cercanos (NIRS, INFRATEC 1241, Plano, Texas, USA), que analiza en conjunto todas las semillas de una mazorca. De las 200 familias seleccionadas en campo se seleccionaron las 50 familias con mayor contenido de aceite $(25 \%$ de presión de selección entre familias); posteriormente, se efectuó una nueva selección con el aparato INFRATEC 1255 para identificar las semillas individuales con mayor contenido de aceite dentro de cada una de las 50 familias seleccionadas. Los resultados de la respuesta a la selección de estas poblaciones se consignan en PreciadoOrtiz et al. (2013) y Ortega-Corona et al. (2015).

Las LDH progenitoras del $\mathrm{H}-388 \mathrm{O}$ se derivaron del séptimo y octavo ciclo de selección recurrente para ACA en PBB y PBN. La formación de las LDH inició en el año 2011 mediante las siguientes etapas: 1) inducción de haploidía mediante la cruza de PBBy PBN con polen del inductor RWS $\times$ UH400, 2) identificación de semilla haploide mediante el marcador Navajo R1-nj, 3) duplicación de los haploides con colchicina como inhibidor mitótico y 4) aumento de semilla de las nuevas LDH por autofecundación (Prasanna, 2012). A partir del ciclo PV 2012 se llevó a cabo el incremento y selección per se y por cruzamientos de las nuevas LDH en el CEBAJ.

El progenitor femenino del $\mathrm{H}$-388 O tiene alto potencial de rendimiento, es de porte alto, ciclo intermedio, mazorca larga, con 16 a 18 hileras de granos semidentados de color blanco cremoso y forma de la corona hendida. El progenitor masculino es de ciclo intermedio, porte mediano, mazorca mediana con 14 a 16 hileras, grano semidentado color blanco cremoso, forma de la corona hendida, espiga mediana, compacta y alto número de ramas principales.

El H-388 O tiene un buen potencial de rendimiento, tolera el acame, la mazorca presenta buena cobertura, presenta un ciclo de madurez intermedio, porte alto, espiga larga, alta presencia de ramas laterales, mazorca sana de tamaño medio, con 18 a 24 hileras de granos, ligeramente en espiral, color blanco cremoso, textura semidentada y forma de la corona hendida (Figura 1).

El H-388 O se evaluó durante los años 2014, 2015 y 2016 en ocho ambientes subtropicales, con un rendimiento de grano promedio de $12.6 \mathrm{t} \mathrm{ha}^{-1}$ (intervalo entre 7.0 y 16.2 t ha $^{-1}$ ), floración masculina de 68 a 80 d y femenina de 70 a $80 \mathrm{~d}$; altura de planta de 170 a $355 \mathrm{~cm}$ y de mazorca de 83 a $200 \mathrm{~cm}$. Al comparar el rendimiento y características físico-químicas del H-388 O contra el testigo $\mathrm{H}-377$ en ocho ambientes, el H-388 O mostró mayor rendimiento y contenido de aceite, así como mayor índice de flotación; sus granos fueron más pequeños (<PCG) y el peso hectolítrico, contenido de almidón y proteína fueron menores que en el testigo (Cuadro 2).

Este híbrido se registró en el año 2018 en el Catálogo Nacional de Variedades Vegetales (CNVV) del Servicio Nacional de Inspección y Certificación de Semillas con la clave MAZ-1854-090318 y con título de obtentor número

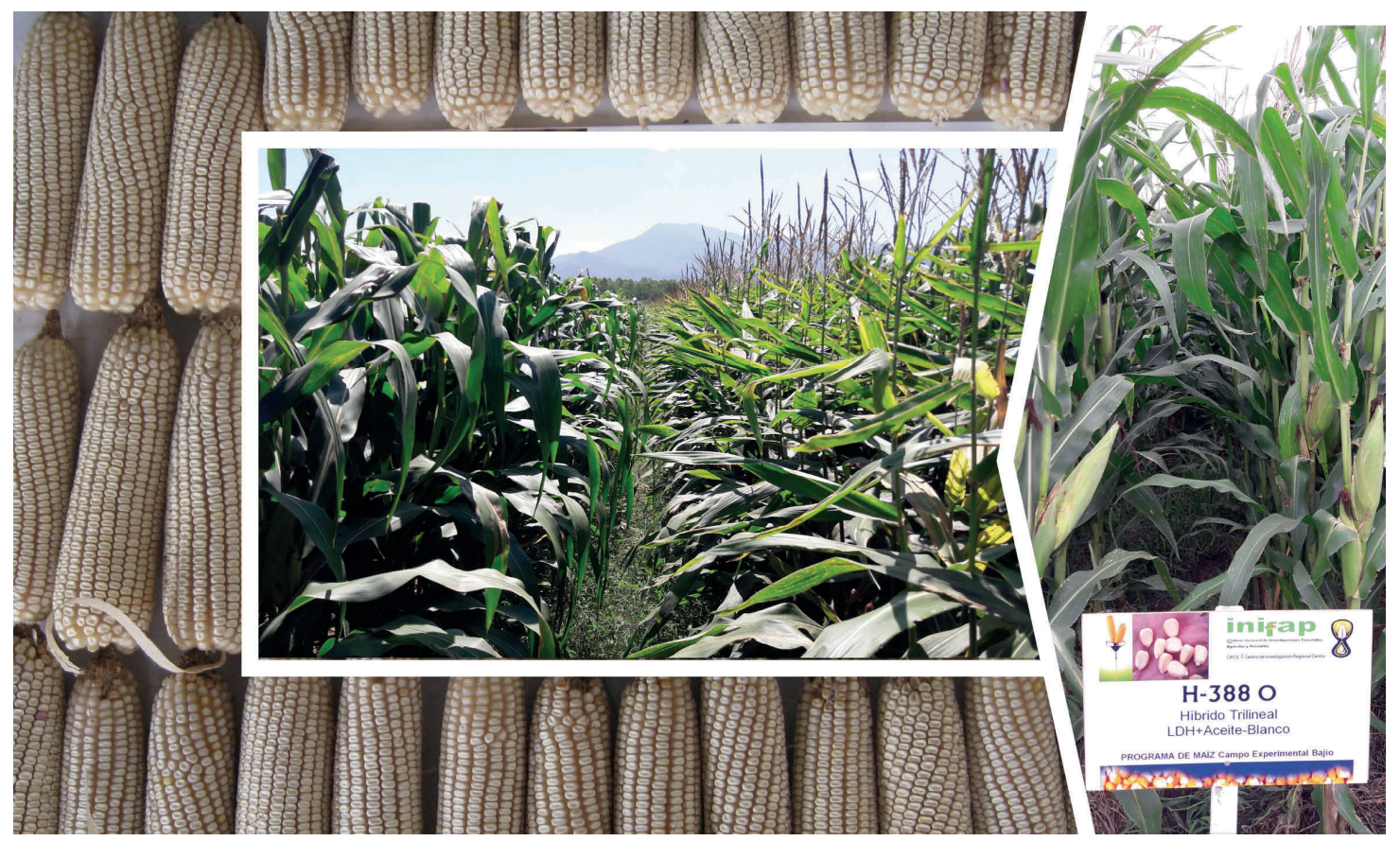

Figura 1. Características de planta y de mazorca del híbrido de maíz blanco oleoso H-388 0. 
Cuadro 2. Medias del rendimiento y características físico-químicas del híbrido $\mathrm{H}-388 \mathrm{O}$ de grano blanco oleoso en ocho ambientes subtropicales ${ }^{\dagger}$. Primavera-Verano 2014-2016.

\begin{tabular}{lccccccc}
\hline Genotipo & $\begin{array}{c}\text { Rendimiento } \\
(\mathrm{t} \mathrm{ha-1})\end{array}$ & $\begin{array}{c}\text { Índice de } \\
\text { flotación (\%) }\end{array}$ & $\begin{array}{c}\text { Peso de } 100 \\
\text { granos }(\mathrm{g})\end{array}$ & $\begin{array}{c}\text { Peso } \\
\text { hectolítrico } \\
\left(\mathrm{kg} \mathrm{hL}^{-1}\right)\end{array}$ & $\begin{array}{c}\text { Contenido de } \\
\text { aceite (\%) }\end{array}$ & $\begin{array}{c}\text { Contenido de } \\
\text { almidón (\%) }\end{array}$ & $\begin{array}{c}\text { Contenido de } \\
\text { proteína (\%) }\end{array}$ \\
\hline $\mathrm{H}-388 \mathrm{O}$ & $12.86 \mathrm{a}$ & $30.16 \mathrm{a}$ & $31.59 \mathrm{~b}$ & $77.50 \mathrm{~b}$ & $6.54 \mathrm{a}$ & $66.72 \mathrm{~b}$ & $8.93 \mathrm{~b}$ \\
$\mathrm{H}-377$ & $10.05 \mathrm{~b}$ & $15.63 \mathrm{~b}$ & $35.23 \mathrm{a}$ & $78.51 \mathrm{a}$ & $5.07 \mathrm{~b}$ & $69.10 \mathrm{a}$ & $9.49 \mathrm{a}$ \\
DMS & 1.87 & 2.17 & 0.62 & 0.72 & 0.23 & 0.65 & 0.32 \\
\hline
\end{tabular}

†: Ambientes de prueba Celaya, Gto. $20151^{\text {a }}$ fecha; Celaya, Gto. $20152^{\text {a }}$ fecha; Pabellón, Ags. 2015; Tarimbaro, Mich. $2015 ;$ Celaya, Gto. 2016 $1^{\text {a }}$ fecha; Celaya, Gto. $20162^{\text {a }}$ fecha; Tarimbaro, Mich 2016; y Juventino Rosas, Gto. 2016. Medias con letras iguales en las columnas no son estadísticamente diferentes (DMS, $\mathrm{P} \leq 0.05)$.

2044. La semilla de categoría básica del progenitor masculino (LBDHBO 3) y de categoría registrada del progenitor femenino (LBDHBO $1 \times$ LBDHBO 2) está disponible en el CEBAJ, previa solicitud al INIFAP, para los interesados en la producción de semilla certificada del $\mathrm{H}-388 \mathrm{O}$.

\section{BIBLIOGRAFÍA}

Alexander D. E. (1988) Breeding special nutritional and industrial types. In: Corn and Corn Improvement. G. F. Sprague and J. W. Dudley (eds.) Third edition. American Society of Agronomy. Madison, Wisconsin, USA. pp: 869-880.

Lambert J. R. (2000) High-oil corn hybrids. In: Specialty Corns. A. R. Hallauer (ed.). Second edition. CRC Press. Boca Raton, Florida, USA. pp:131-154.
Ortega-Corona A., R. Picón-Rico, R. E. Preciado-Ortiz, A. D. Terrón-Ibarra, M. J. Guerrero-Herrera, S. García-Lara and S. O. Serna-Saldivar (2015) Selection response for oil content and agronomic performance in four subtropical maize populations. Maydica 60:1-8.

Prasanna B. M. (2012) Doubled haploid (DH) technology in maize breeding: an overview. In: Doubled Haploid Technology in Maize Breeding: Theory and Practice. B. M. Prasanna, V. Chaikam and G. Mahuku (eds). CIMMYT. México, D. F. pp:1-8.

Preciado-Ortiz R. E., S. García-Lara, S. Ortiz-Islas, A. Ortega-Corona and S. O. Serna-Saldivar (2013) Response of recurrent selection on yield kernel oil content and fatty acid composition of subtropical maize populations. Field Crops Research 142:27-35, https:// doi.org/10.1016/j.fcr.2012.11.019

Vázquez-Carrillo M. G., R. E. Preciado-Ortíz, D. Santiago-Ramos, N. PalaciosRojas, A. Terrón-Ibarra y A. Hernández-Calette (2018) Estabilidad del rendimiento y calidad de grano y tortilla de nuevos híbridos de maíz con valor agregado para el subtrópico de México. Revista Fitotecnia Mexicana 41: 509-518. 
